\title{
Témoignage : Henry Bauchau aux sources de la création poétique. Éveil au poème, don du rêve
}

L’Université de Cergy-Pontoise a le plaisir d'accueillir Monsieur Guy Samama, directeur de la rédaction de la revue Approches depuis 2010, dont le dossier du n¹52 de décembre 2012 est consacré à Henry Bauchau. Guy Samama est agrégé de philosophie, il a été durant huit années conseiller auprès du président du Collège international de philosophie, professeur dans plusieurs lycées et à l'Université d'Amiens. Il est également le créateur en 1976 de L'Avant-Scène Opéra. Il a édité plusieurs ouvrages philosophiques liés notamment à l'éthique et à la médecine. Parmi les parutions récentes, signalons chez François Bourin D'un prétendu droit de mourir par humanité de Bernard-Marie Dupont paru en 2011 et L'Unité cachée : judaïsme, christianisme, islam d'Antoine Schwarz paru en 2012. Guy Samama est l'auteur de plusieurs livres para-universitaires, parus chez Ellipses, à destination des étudiants de philosophie. Il collabore à la revue franco-portugaise Sigila qui s'articule autour de la problématique du secret, ainsi qu'à la revue Esprit. Il a été amené à animer dans le cadre de l'AFAR une formation d'art thérapie pour des aides soignantes dont une des étapes était articulée autour de L'Enfant bleu. Ainsi est-il entré en consonance avec cette attention à l'autre, cette voix intérieure, et le désir d'écoute qu'il reconnaît dans l'écriture d'Henry Bauchau.

La revue trimestrielle de sciences humaines Approches dont il s'occupe actuellement a été créée en 1974 par le Père Plusse: le sous-titre "Questions sur l'homme. Questions sur Dieu» porte la trace de cet héritage religieux mariste. Initialement publiée sous la forme d'un bulletin d'accompagnement en direction de personnels soignants, d'éducateurs spécialisés, et de la petite enfance, Approches s'affirme aujourd'hui comme une revue de réflexion et d'analyse désireuse de prendre du recul par rapport aux différents débats contemporains qui l'intéressent concernant aussi bien la politique, la santé, la psychanalyse, 
l'éducation et la littérature à travers des numéros thématiques et d'autres consacrés à des auteurs comme Albert Camus, Simone Weil, Henry Bauchau et Stefan Zweig.

Le texte qui suit est issu d'une intervention orale au colloque Henry Bauchau des 12 et 13 juin 2013, organisé par Catherine Mayaux et Olivier Belin à l'Université de Cergy-Pontoise. Nous lui avons conservé son style personnel, volontairement kaléidoscopique et «impressionniste» surtout dans la première partie. Cet écrit ne se veut donc ni théorique ni non plus analytique.

\section{Contradictions et métamorphoses}

Puisque vous avez bien voulu, chère Anne-Sophie Miccio, m'interroger sur ce qui m'attire, et en même temps me trouble, chez Henry Bauchau, je vais essayer de vous répondre en démêlant, en dépliant les écheveaux dans lesquels je suis plié et emmêlé.

- Une écriture qui n'est pas tournée vers soi, mais d'abord vers les autres. Presque thérapeutique avant la lettre, ou par la lettre. Attentive au son autant, sinon plus, qu'au sens grâce à une oreille innocente. Paradoxe : tournée vers le dehors (autrui aussi bien que la Nature «Les arbres agissent sur nous plus par nos perceptions inconscientes que par la vue. Nous sommes en eux [...]»(PBG, p. 275), elle creuse le sillon d'un chemin vers une liberté intérieure. Elle est son «soleil intérieur» ( $J A$, p.311). Elle est « une vraie forme de générosité » (Ibid., p. 308);

- Un voyage à travers l'Histoire du $\mathrm{XX}^{\mathrm{e}}$ siècle : Henry Bauchau a été contemporain des deux plus grandes guerres de l'humanité, et subi deux traumatismes; dans la première, c'est celui de l'incendie de Louvain alors que, séparé de ses parents, surtout de sa mère (la séparation d'avec sa mère pendant trois mois à l'âge de dix-huit mois en 1914, première blessure, d'où naîtra Mérence $=$ mère + absence) ; il était chez ses grandsparents maternels; c'est avec eux et une jeune servante qu'il franchit à l'aide d'une petite échelle les murs des jardins au milieu de maisons en flammes: nuit d'épouvante. Lors de la deuxième guerre, ce furent son engagement dans le mouvement des VT (Volontaires du Travail), et le malentendu qui s'ensuivit puisque la propagande a fait dévier les visées de ce projet en répandant la rumeur que les VT étaient des collabos, et qu'il reçut de la part d'une assemblée d'officiers dirigée par un général un 
blâme pour conduite antipatriotique : «Une sentinelle me demande mon nom et, quand je signale que nous sommes des hommes de l'escadron Brumagne, j'entends un des résistants dire: "Bauchau... Bauchau ... Mais ce sont les VT ! Ce sont des collabos! On ne peut tout de même pas laisser entrer tout le monde dans la Résistance." Je suis profondément blessé mais je n’en laisse rien voir » ${ }^{1}$.

Mais il y aurait bien d'autres raisons :

- parce qu'il n'est pas dans la réconciliation. Il est dans la déchirure. Il est un être séparé ;

- parce que, selon le mot d'un ami médecin, il est un être double, et que ses tentatives pour le réduire à l'unité sont douloureuses; il y aurait deux Bauchau comme il y a deux avant-guerres, deux après-guerres : deux îles secrètement reliées sous les eaux ;

- parce qu'il a commencé à écrire des romans à l'âge de 45 ans, et qu'il écrivit de nombreuses versions successives de la plupart de ses textes ;

- parce qu'il a connu deux psychanalystes, dont l'une, durant sa première vie, Blanche Jouve Reverchon appelée par lui la Sibylle, est devenue à la fois figure tutélaire, Pythie et amie ;

- parce que psychanalyse et écriture ont convergé chez lui au point d'être difficilement séparables. L'écriture est un prolongement de l'analyse par d'autres moyens. Elles agissent, réagissent, évoluent ensemble. L'une nourrit l'autre, et s'en nourrit.

«Écriture et psychanalyse mêlent leurs espérances et leur nécessité » (EE, p. 121). Par exemple, parlant de La Déchirure, tentative d'exutoire au traumatisme de la mort de sa mère, et de son origine lointaine, il écrit : «Ce travail, que je ne considère pas comme une entreprise d'écriture, mais seulement comme un fragment d'analyse [...]. Il y a près de dix ans que j'ai terminé l'analyse, je songe à dire ce qu'elle est devenue dans l'écriture qui, comme l'analyse, va vers le nouveau, le caché » (Ibid., pp. 51-52). L'analyse poursuit un originel passé, l'écriture le reprend en le transformant en originel futur ;

${ }^{1}$ Henry Bauchau, Chemin sous la neige, Actes Sud, 2013, p. 128 ; voir aussi p. 153. 
- parce que la Chine a tenu une place décisive dans son imaginaire, Chine intérieure, lieu où les destructions sont toujours suivies de renaissances ;

- parce que j'aime en lui l'alliance entre la Grèce et la Chine, entre Diotime et Lao-tseu. La Grèce, c'est la naissance de la philosophie, la lumière du logos; la Chine, c'est l'absence de besoin du discours : il se dissout dans la sagesse.

- parce que j'aime en lui la dialectique entre la force et la faiblesse : "Ta "force faible" te fait avancer comme une eau que rien n'arrête » ( $J A$, p. 243), lui écrivait Robert Dreyfuss parlant notamment de Jour après jour. Bauchau se sent constamment faible, fragile, en échec, en lutte contre le doute et l'incertitude, surtout après la disparition de Laure le 27 juin 1999. En même temps, il déploie une énergie décuplée, avec des forces renouvelées, dès qu'il s'agit de lire, et surtout d'écrire. "Quant à moi, j'ai appris à écrire seul, en lisant beaucoup, en faisant, en refaisant, en luttant contre le doute et en demeurant presque toujours dans une sorte d'incertitude» (PBG, p. 187). Ce sentiment d'être toujours dans l'ombre, toujours second: «Toujours l'image du second en moi ». Dès l'enfance, L'Enfant rieur pleure souvent parce qu'il se sent inférieur à son frère aîné Jean (Olivier dans L'Enfant rieur), et humilié par les enfants. Encouragé comme son frère et ses cousins à sauter par-dessus une rivière à la perche, il tombe à l'eau. Il croyait être devenu à leurs yeux «celui qui tombe dans la rivière $»^{3}$. Il a serré les dents malgré les moqueries des autres.

- parce qu'il est l'homme des métamorphoses, non pas seulement celles qu'il fait subir à Antigone par le passage de la troisième à la première personne, mais celles qu'il constate en lui sans que sa volonté y prenne part :

Dans La Grande Troménie, je dis en somme qu'il faut s'abandonner à la métamorphose. Je pensais alors et je pense toujours qu'il faut prendre le risque des grandes métaphores de l'existence et des choses que nous pouvons réaliser avec nos propres forces. La métamorphose, elle, suppose un changement dont nous ne sommes plus les pilotes. Nous

\footnotetext{
2 Ibid., p. 262.

${ }^{3}$ Henry Bauchau, L'Enfant rieur, Actes Sud, 2011, p. 36.
} 
agissons encore, mais en étant agis dans nos profondeurs. Le dessein n'est plus le nôtre, il est celui d'un ensemble, d'un rien, d'un tout dont les mécanismes et la grâce sont hors de notre portée. Comme pour le poème peut-être, notre rôle n'est plus de vouloir, mais seulement de lutter contre la résistance. ( $J A, \mathrm{p} .429$.)

Me frappe par son acuité cette notation psycho-sociologique :

Le fait qu'à l'explosion des pouvoirs de l'homme dans le monde occidental répondent des sociétés psychologiquement dépressives [...] est le phénomène peutêtre le plus marquant de notre époque depuis la fin des trente glorieuses. (PBG, p. 197.)

Ce déséquilibre entre la recherche capitaliste du rendement, des plaisirs immédiats, de l'efficacité et de la rapidité d'un côté, de l'autre les malheurs, les violences, les souffrances, les dépressions qui affectent un nombre croissant de nos contemporains, s'impose comme un contraste à analyser et à interpréter :

Tout m'incite en somme à me consacrer toujours plus à l'écriture et à la prière. Dans le dangereux glissement du monde vers l'assouvissement des désirs, la consommation, la technique, je puis à ma petite place rester fidèle à la vie intérieure et à la vérité artisanale de l'art vécu comme un chemin. (Ibid., p. 391.)

Ce qui importe, c'est le chemin, non le but; le cheminement dans les labyrinthes d'écriture. Un chemin délivré non des contradictions, mais de l'angoisse qui leur est le plus souvent attachée. Antigone et CEdipe sont ses compagnons de route. Ils ne savent pas où ils vont, mais ils savent qu'ils doivent aller - aller vers l'inconnu. "La route est une image de l'état de mobilité intérieure » $(J A$, p. 306) ; "Antigone (à Créon) : "Ceux qui sont en place connaissent leur lieu de vie, leur travail. Ceux qui sont en marche ne connaissent que la route" (I Ibid., p. 268) - un ancien patient lui écrivait : "Je ne suis pas arrivé (où)».

- parce que l'écriture, le travail de l'écriture, provient chez lui d'une obscurité initiale, et d'une inhibition d'écrire : 
Pourquoi cette résistance acharnée à l'écriture ? Peut-être parce qu'il y a une secrète interdiction à cette marche indirecte vers le sacré. Le sacré exige tout et l'écriture ne lui donne pas ce tout, elle en garde une partie pour l'art, les jouissances de la forme et celles du narcissisme. L'écriture aussi prélève par la sublimation une part de la pulsion sexuelle, celle-ci résiste. » (Ibid., p. 457.)

En même temps, tout ce qui le détourne de l'écriture est ressenti par lui comme un vide "Une journée sans écrire est devenue pour moi une journée affligée par un sentiment de vide» (Ibid., p. 360).

- parce que le désir du poème nait dans la douleur, de la douleur, et que lui n'était armé que du sabre de la petite enfance, de son éclat passager comme celui brandi par son frère aîné aux cheveux blonds Olivier, prince oral dans la plénitude de lui-même et du monde, relié à la terre mère, devenu fermier et pasteur de troupeaux :

Le poète mal armé pour faire face à la grande vieillesse. Je ne suis pas sans armes, mais peut-être mal armé pour la vie telle qu'elle est. Mon père, lui, était armé par l'argent qui me manque. (Ibid., p. 349.)

- parce que ce père voulait être officier, que sa famille l'en a empêché, qu'il ne s'est pas rebellé et qu'Henry Bauchau en a porté toute sa vie l'humiliation et la honte : «mal armé ».

- parce que, dans ses livres, il s'abandonne à ses rêves, à ses fantasmes, au peuple des pulsions. Appartenant à ce peuple, il fait signe vers la lumière. Il appartient aussi au peuple du désastre: celui des blessés et des handicapés psychiques ;

- parce qu'il écrivait sous dictée intérieure et suivant l'enchaînement des associations, comme Orion dans ses dictées d'angoisse à Véronique, à la fois psychanalyste et artiste, un peu à son image.

- parce qu'il a conduit certains de ses patients de la psychanalyse vers l'écriture, et que ceux-ci sont devenus écrivains ou analystes-écrivains. Il est frappant de constater que ceux qui furent ses patients en analyse sont devenus des partenaires en écriture, comme si l'écriture était un moyen de prolonger l'analyse. Ainsi Chantal Deltenre, ethnologue et écrivain, écrit dans son journal : 
Quand je lui confie mon désarroi, Henry Bauchau dit: Vous avez vécu jusqu'ici en grand arroi. Il faut accepter le désarroi. C'est un cycle. Seuls les Chinois comprennent cela. Je m'accroche au dessin derrière lui, j'essaie de faire mienne la légèreté de ce cheval dansant. Vous commencez un gros travail en entamant une thérapie. C'est épuisant de garder ainsi l'esprit en alerte, dit le thérapeute. C'est le conseil de l'écrivain que j'entends. Garder l'esprit en alerte, apprendre à voir, à saisir l'instant, c'est cela écrire. C'est ainsi qu'il écrit. ${ }^{4}$

Nous frappent plusieurs points : le diagnostic analytique porté par Henry Bauchau sur sa patiente, transformé par celle-ci, qui cherche à devenir écrivain, en conseil d'écrivain; la mention des chevaux, dont nous savons combien Henry Bauchau les appréciait, lui-même ayant été un bon cavalier; la légèreté d'un cheval dansant, auquel sa nouvelle patiente tente de s'identifier: légèreté des pulsions? Cette écriture voudrait-elle signifier, qu'initiatique en même temps qu'analytique, elle doit affronter un rapport douloureux à l'échec toujours à nouveau possible?

Tous ces « parce que » n'expliquent rien.

\section{Une voix profonde venue d'ailleurs: naissance au poème}

Pour moi, la vraie raison, qui m'a fait m'intéresser à lui, est qu'il était poète à la racine. Son inconscient l'était naturellement. L'ensemble de son œuvre, pas seulement la poésie, dérive de cette source. «L'ensemble de mon œuvre est un poème, mais le poème rythmé, lié à la musique intérieure de la langue, doit être chargé d'une énergie particulière. D’une invention qui l'assimile plus à une œuvre de la nature qu'à une action volontaire $»^{5}$.

La poésie comme langue naturelle initiale, voire initiatrice, est ce qui irrigue toute sa pensée: source vive d'avant mémoire. Elle irrigue une végétation foisonnante intérieure. «Le lieu du poème n'est pas dans le

\footnotetext{
4 Approches, Henry Bauchau, Inédits, correspondances, hommages, numéro spécial, nº152, décembre 2012, p. 128.

${ }^{5}$ Ibid., p. 174.
} 
délire car le délire ne veut pas s'astreindre à la forme et à la transmission, il veut exploser, se déployer sans frein. Le poème doit frapper à la porte du délire, ne pas entrer mais recevoir les dons que le délire, le rêve, les pulsions font à ceux qui sont prêts pour cela. C'est dans cette innocence de l'oreille, prête à tout écouter, à tout peser et à retenir le meilleur, que se trouve l'acte du poète. Tout le reste est don et grâce »"

Il n'est pas si éloigné de Platon, qui, dans le dialogue Ion, laisse entendre que l'inspiration poétique, proche du délire, est un don des Dieux : «Les poètes ne sont rien que les interprètes des dieux, et chacun d'eux est possédé par le dieu qui s'empare de lui » ${ }^{7}$. Interprètes des dieux chez Platon, des rêves chez Bauchau. Cette inspiration chez Platon, cette innocence de l'oreille chez Bauchau, échappent au contrôle de la raison. Elles sont délirantes, par là même déliantes.

Dans sa poésie, il se met d'abord à l'écoute d'un rythme intérieur, musique qui vient avant les mots. Guidé par un rêve ou par une image, il va vers un certain son de voix, un certain rythme respiratoire. "Hier, moment de joie, en sentant qu'un poème commencé semblait prendre forme, s'orienter vers un son que j'entends un peu mais ne discerne pas encore $»^{8}$. C'est le son qui semble aimanter le poème, non le sens, et ce son n'est pas un but: "Je suis souvent guidé par un rêve, par une image mais rien ne s'accomplit si un rythme n'apparait pas, un chant ou l'ébauche d'un chant » $(J A$, p. 225). La poésie n'est pas orientée par le bien ni par le mal, elle ne l'est pas non plus par le beau qui n'est qu'un de ses chemins, elle est orientée par le nouveau qui est un renouveau et l'àvenir du poème. «Le poème, rythme, mots, musiques, n'est pas destiné à un possesseur ni à une possession» (Ibid., p. 169). Le mot est dans l'oreille enfantine, c'est là qu'il faut aller le chercher pour l'entendre. La poésie est «Exercice du matin », selon le titre d'un de ses poèmes :

Le matin sortant du fleuve

Sortant du lit débordé.

Aventure de la terre

Au regard miraculé

\footnotetext{
${ }^{6}$ Ibid., p. 63.

7 Platon, Ion, 534b-534c, trad. M. Canto-Sperber, Euvres complètes, Luc Brisson (dir.), Paris, Flammarion, 2008, p. 577.

${ }^{8}$ PBG, 29 septembre 2001, pp. 389-390.
} 
Marge du soleil levant

Exercice de l'éveil

Elle est aussi louange car «La poésie est tournée plus directement vers le divin » (Ibid., p. 79).

Elle est rapport de liberté avec «la bouche d'ombre » (Victor Hugo), et charme jeté sur le monstre selon des mots de Pierre Jean Jouve parlant de l'imagination. Instrument de courage et de guérison, le poème est déjà là avant son écriture, de sorte que, dans le labyrinthe, nous allons vers ce qui existe et se dévoile peu à peu. Car le poème ne vient pas d'abord du poète, pas de son désir. Il est appelé et, de ce lieu inconnu, il appelle le poète si son désir parvient à lui répondre. Le poète n'est pas maître chez lui. Il n'a pas de chez lui. Il peut approcher de sa circonstance, de ce qui tourne autour sans se poser, mais il ne l'atteint pas, il en est un traducteur, le poème est appelé à naitre, cet appel ne vient pas de son être. Ce charme que la forme jette sur le monstre engendre un jeu entre l'écriture, le corps, l'image et la musique. De ce jeu, le corps n'est pas absent; d'abord hédoniste, il est devenu peu à peu oblatif; un corps sportif, athlétique, devenu créateur: c'est par lui que Bauchau se rapproche des vérités de la main. Ainsi a-t-il entrepris de dessiner et de peindre, comme son patient psychotique Lionel, Orion dans L'Enfant bleu.

Voici comment il décrit lui-même dans «L'innocence de l'oreille », dernier chapitre de L'Écriture à l'écoute, non la genèse, mais la venue à soi du poème : "Me voilà au point où je dois tenter de vous dire comment j'ai pu écrire des poèmes tout en étant, comme Henri Michaux, incapable, pendant ce temps, de maitrise sur mes pensées et mes affects. Je vais essayer de vous montrer le cheminement, à travers maintes obscurités, d'un de mes derniers poèmes» (EE, p. 151). Suit la description d'une expérience d'éveil, qui convoque comme un appel. Il s'agit du poème Exercice du matin : «Le matin sortant du fleuve /Sortant du lit débordé $[. .$.$] ». Débordé par quoi ? demande-t-il. Débordé par le$ rêve comme par un impératif intérieur. Ce poème doit s'inscrire dans la ligne du premier exercice du matin pour se préparer à la journée, et comme un exorcisme pour conjurer l'événement qui va bouleverser sa vie. Il doit être un instrument de courage et de guérison. Le poème est là, avant son écriture. Il faut donc aller vers ce qui existe, et se dévoile peu à peu. Mais il ne vit pas avant d'être sorti du labyrinthe. S'il a déjà un passé, 
il n'a pas encore de présent ni de futur avant de pouvoir être écrit. «Écriture sur le sable / Tables du sommeil profond ».

Écrire sur le sable, c'est bien ce qu'on vit chaque jour dans le poème: les pensées, les mots, les sons, les images apparaissent, se transforment, s'inversent et s'effacent sans cesse $[. .$.$] . L'exercice du langage, dans la musique corporelle$ du poème, ne peut plus s'exercer que dans l'obliquité du soleil levant ou déclinant, dans la déchirure intime qui est celle de notre temps. (Ibid., p. 154.)

Le poème prend corps, dirions-nous, dans l'effacement des traces de nos actes et de nos pensées; dans l'ubiquité invisible de l'obliquité. Les mots essentiels, il faut les entendre à la porte du délire, entre Dionysos et Apollon. C'est cette obscurité béante, cette obscurité déliante, qu'il doit habiter : lieu sillonné de messages, de sens et de sons. Mais comment les capter? Comment les capter sans les capturer, ce qui reviendrait à les rendre prisonniers en se faisant soimême prisonnier?

Comment capter les couleurs insensées, les sons célestes ou sauvages de l'imagination profonde ? C'est là que le poème s'arrête, qu'il sonde longtemps et longtemps ne trouve pas. On croyait que c'étaient les mots, ces grands démons de sel, qui devaient écouter à la porte du délire. Mais les mots ne sont pas assez originels. Il faut attendre l'annonciation patiente, celle qui sait que dans le poème c'est l'oreille d'abord qui perçoit. Pourquoi l'oreille ? Parce que c'est elle qui, déjà dans le ventre maternel, entend le bruit, le rythme fondamental du cœur et les premières voix, celle de la mère et celle du père. C'est donc l'innocence de l'oreille qui peut entendre ce qui n'a pas encore été entendu et se prosterner plus profond. (Ibid., p. 155.)

Dans ce texte si riche, prélevons les éléments suivants : d'abord, les mots s'ils se présentent en premier sont les mauvais démons du poème. Ils ne sont pas assez premiers. Ils devraient être matière en fusion perpétuelle. Ensuite, les termes annonciation et se prosterner nous font entendre une résonance sinon mystique, du moins religieuse, ou bien 
ouvrant sur l'espérance. Enfin, et surtout, l'expérience du poème est semblable à celle de la naissance. C'est une expérience du seuil, d'un passage. Du bord du gouffre, surgit de l'être. Or, la poésie, comme la naissance, est fondatrice. Nous avons à faire alliance avec elle pour créer un lieu qui soit à la fois insondable et habitable. Loin de toute construction conceptuelle, la poésie est une voix qui monte du corps. Elle est en avant et en arrière de toute tentative de saisir la vérité. Mais tout peut s'effondrer : nous sommes alors dans le désastre.

\section{Corps musical, rêve, écriture}

Vous m'avez posé, Anne-Sophie Miccio, une deuxième question: parmi les inédits que je publie dans le numéro spécial 152 «Henry Bauchau, Inédits, correspondances, hommages » de la revue Approches, paru en décembre 2012, quels sont ceux que je préfère? Question difficile, car elle suppose un choix. Or, celui-ci a été effectué en amont, avant publication. N'ont donc été retenus que les textes déjà sélectionnés.

Mais je vais y répondre le plus honnêtement possible, au risque de commettre des injustices. J'y répondrai en privilégiant ceux qui engagent moins leurs auteurs qu'ils n'éclairent la démarche de pensée d'Henry Bauchau lui-même.

Tout d'abord, une lettre à François Devenne, géographe et romancier ayant vécu au Kenya, qui fut analysé très jeune sur les conseils de sa mère. Bauchau lui écrit le 13 août 1990 : «Préoccupe-toi du style, de l'humble orthographe, de la concordance des temps (si analytique) et des possibilités de la syntaxe dans l'écriture et la vie »". Ces quelques mots, qui pourraient sembler anodins, dénotent chez Bauchau à la fois un grand souci du style, le lien quasi ombilical qu'il établissait entre l'écriture et la psychanalyse, une recherche de cohérence dans la vie comme dans l'écriture. Car la syntaxe n'est rien d'autre que cet art de la cohérence dans les enchaînements. Cette écriture efface la frontière entre le vécu et le fictionnel, entre le rêve et la vie, entre le rêve et le poème; de sorte qu'Henry Bauchau, non seulement puise dans les mythes des ressources pour l'imaginaire, mais se constitue en mythe. Quand il écrit «mon personnage », il s'agit d'une «crase » entre lui et le personnage mis en

${ }^{9}$ Approches, op. cit., p. 106. 
scène à cet instant. Le rêve, pourrait-on dire, dessine les lignes de ce que la vie doit être en advenant à elle-même dans la sérénité du jour. «Produisez vos rêves » a été l'injonction initiale et initiatrice de la Sibylle. Mais il s'est bien gardé de tomber dans le piège de l'interprétation des rêves.

Dans cette même lettre à François Devenne, Henry Bauchau lui conseillait : «Il faut que tu parviennes à écouter ton corps comme ta flùte [...] En ce qui concerne l'écriture, ne cherche pas l'originalité, laisse-la te trouver. Il y faut du temps car l'original est en réalité l'originel, l'inconscient. [...] Un conseil : ne cherche pas à analyser un thème avant de l'écrire. Laisse "agir» l'inconscient sans le fardeau d'une interprétation préalable $»^{10}$. Dans cette lettre, trois traits font plus spécialement saillie : le corps entendu comme instrument de musique, comme déjà musical ; l'originalité comme propriété de l'origine, qui est l'inconscient; s'évader de la prison de l'interprétation, comme ailleurs Bauchau recommande en rêve de s'échapper de la prison de la rationalité. C'est sans doute la même.

«En rêve je dis : il faut sortir de la prison de la pensée rationnelle. J'ajoute : sortir de l'abîme de la pensée rationnelle » ( $J A$, p. 235). Cette substitution d'un mot à l'autre, d'《 abîme » à "prison » n'est sans doute pas indifférente: dans une prison, nous sommes enfermés, mais des murs existent, un espace, bien que clos ; à l'inverse, dans un abîme nous nous perdons, il n'y a pas de fond, pas de délimitation ni de cloisonnement. La pensée rationnelle, loin de donner des limites à la pensée, l'égarerait. Ce jeu de substitution n'est pas indifférent non plus si nous l'interprétons comme ce que dicte le rêve, ou l'inconscient. «Polynice : l'âge d'or. Étéocle : l'âge d'argent. Leur conflit : l'âge de fer. Pour l'âge d'or, j'ai fait le lapsus calami d'écrire l'âge dort. L'âge où l'on dort encore dans le ventre maternel» (Ibid., p. 130). Il y revient autrement plus loin : «Polynice, l'or, son brillant. Étéocle, l'argent, son caractère sombre. La capacité de l'argent à produire de l'argent par le travail des autres» (Ibid., p. 236). Au lieu que le réel soit modèle pour l'image, un peu comme chez Platon, c'est l'image qui s'érigerait en modèle du réel chez Bauchau : désir des rêves, désir des mots.

${ }^{10}$ Ibid., pp. 106-107 ; nous soulignons. 
«Le désir du rêve ne semble pas le passage d'une biche ou d'une femme réelle. Il est seulement le désir des mots : le passage de la biche » (Ibid., p. 304 ; nous soulignons). Le réel vient en deuxième, ou troisième position, après l'image, après le mot.

Dans une deuxième lettre, du 27 décembre 2007, à un autre écrivain, et chercheur, Olivier Ammour-Mayeur, Henry Bauchau reconnait une dette à l'égard du surréalisme, mais surtout à l'inconscient et à la jeunesse :

Je me suis retrouvé à l'écart de tous les mouvements littéraires d'avant-garde, même si j'ai subi une certaine influence du surréalisme qui est, plus qu'une école littéraire, une façon purement artistique d'aborder l'inconscient [...] ma première pièce Genghis Khan, qu'on vient de redonner à Paris avec un certain succès, écrite alors que j’avais déjà 41 ans, a été une explosion à la fois de l'inconscient et de la jeunesse, tous deux refoulés. ${ }^{11}$

Relevons deux caractères: la mention du surréalisme, expression artistique de l'impulsion et de l'impatience du désir, automatisme de la langue des pulsions; l'explosion de l'inconscient et de la jeunesse, tous deux conjoints, tous deux refoulés. Pourquoi refoulés ? Sans doute parce qu'ils désirent s'assouvir violemment, jouir, crier, hurler, danser, faire l'amour.

Je voudrais, enfin, mentionner un témoignage d'une amie proche d'Henry Bauchau, Marie-Claire Boons :

Henry, le passeur, celui qui ouvre la possibilité de passer d'une rive à l'autre. Celui qui ouvre le cœur et fait pleurer toute une nuit une jeune femme lisant le dialogue d'Antigone et de sa sœur [...] Ce n'est pas tant que la lecture de L'Enfant bleu me transporte, c'et plutôt qu'elle me transforme [...] Ton roman est celui d'une « civilisation des pulsions » : non point pour devenir «normal» mais pour inscrire dans une société cela qu'on peut y devenir, selon la vérité qui vous marque. ${ }^{12}$

\footnotetext{
${ }^{11}$ Ibid., p. 233.

12 Ibid., pp. 197-198.
} 
Passer d'une rive à l'autre - c'est ainsi que Bauchau parlait de la mort de Laure : elle est passée sur l'autre rive. Les effets thérapeutiques de la lecture d'un récit thérapeutique - L'Enfant bleu - sont aussi évoqués. Marie-Claire Boons termine son témoignage par ces mots :

Il y a que les poèmes d'Henry, au fur et à mesure des années, vont en se dépouillant à l'extrême jusqu'à tenir dans quelques mots le pire, jamais démenti, et l'émerveillement, toujours là :

Dans le champ du malheur

les grands apprentissages

plantent toujours

leur objection

Mais aussi : branches émerveillées

Avant la fin de la lumière

«Brèves intermittences » écrira Henry, qui lui sont données au cœur d'un travail sans trêve, murmures innombrables, déployant en chacun de nous une immensité paisible, l'existence comme la possibilité d'une fête. ${ }^{13}$

L'intégralité de ce témoignage émouvant serait à lire. Relevons seulement cette fête de l'existence, que Bauchau célèbre si souvent, surtout quand il se sent vieillir. Relevons aussi que le processus d'écriture consiste chez lui à enlever la graisse des mots, à élaguer à partir d'une surabondance jusqu'à trouver le mot et le ton justes. Belle image de Myriam Watthee-Delmotte dans son essai sur Bauchau: "Un texte d'Henry Bauchau se reconnait comme une silhouette de Giacometti : y a disparu tout ce qui n'est pas nécessaire. Reste l'ossature tremblée ${ }^{14}$.

"L'à quoi bon, s'il ne désespère pas, élague » (PBG, p. 96). Toujours, chez lui, cette proximité du gouffre, et ce sentiment que rien n'est achevé, qu'il faut retravailler sans cesse. Nous pensons aussi à Simone Weil, qui a eu une grande influence sur sa pensée en l'orientant vers le religieux et la pensée mystique: la création n'est pas dans une surabondance, mais dans un retrait. Le Créateur se retire de sa création.

\footnotetext{
${ }^{13}$ Ibid., pp. 199-200.

${ }^{14}$ Myriam Watthee-Delmotte, Henry Bauchau. Sous l'éclat de la Sibylle, Actes Sud, 2013, p. 161.
} 
Droit $d u$ rêve, droit au rêve et droit de retrait se font écho.

En conclusion, nous citerons ces mots du Journal d'Antigone: "Je commence La Vie des abeilles de Maeterlinck. Ce livre est sans doute dépassé sur le plan scientifique, il ne l'est pas en tout cas sur celui de la vérité poétique » $(J A, \mathrm{p} .24)$. Nous les citons non pas pour évoquer les qualités du critique littéraire, mais pour souligner que, peut-être, la grande affaire de Bauchau est de parvenir à la vérité poétique. Elle a sa source avant la pensée. Celle-ci ne fait que la rejoindre.

Acceptation, espérance et révolte. Révolte par l’espérance dépassée.

Guy SAMAMA

Directeur de la rédaction de la revue Approches Propos recueillis par Anne-Sophie MICCIO Université de Cergy-Pontoise 